\title{
Non-contact bimodal magnetic force microscopy
}

\author{
J. Schwenk, ${ }^{1, a)}$ M. Marioni, ${ }^{1}$ S. Romer, ${ }^{1}$ N. R. Joshi, ${ }^{1}$ and H. J. Hug $^{1,2}$ \\ ${ }^{1}$ Empa, Swiss Federal Laboratories for Materials Science and Technology, CH-8600 Dübendorf, Switzerland \\ ${ }^{2}$ Department of Physics, University of Basel, $\mathrm{CH}-4056$ Basel, Switzerland
}

(Received 18 December 2013; accepted 6 March 2014; published online 20 March 2014)

\begin{abstract}
A bimodal magnetic force microscopy technique optimized for lateral resolution and sensitivity for small magnetic stray fields is discussed. A double phase-locked loop (PLL) system is used to drive a high-quality factor cantilever under vacuum conditions on its first mode and simultaneously on its second mode. The higher-stiffness second mode is used to map the topography. The magnetic force is measured with the higher-sensitivity first oscillation mode. (C) 2014 AIP Publishing LLC. [http://dx.doi.org/10.1063/1.4869353]
\end{abstract}

Magnetic Force Microscopy is a well-established tool to map stray fields emanating from domain structures in a magnetic sample. It can reach spatial resolutions down to $10 \mathrm{~nm}$ with a sensitivity sufficient to detect fractions of a monolayer of uncompensated pinned spins at ferromagnet/antiferromagnet interfaces. ${ }^{1}$ Most magnetic force microscopes (MFMs) use a two-passage method, where each line is scanned in a intermittent contact mode to measure the topography of the sample and subsequently with the tip lifted off the surface to record the magnetic signal. ${ }^{2,3}$ This measurement mode is robust and well applicable to samples with arbitrary topography, e.g., rough samples or patterned media. However, the intermittent contact mode is difficult to apply in vacuum required for high lateral resolution MFM as outlined in detail below. Recently, Li et al. ${ }^{4}$ reported a single passage method that makes use of bimodal cantilever excitation introduced by Rodriguez and Garcia. ${ }^{5}$ The cantilever was driven on its first mode at an amplitude of several tens of nanometers and simultaneously on its second mode at a much smaller amplitude. The first mode amplitude was kept constant by the feedback that controls the tip-sample distance, i.e., reflects the measured topography of the sample. The second mode amplitude and phase were used to record the magnetic signal. Note that for a scan of this kind to be carried out in a reasonable amount of time the oscillation amplitude must stabilize quickly at every point. Hence, very high cantilever quality factors are impractical. Therefore, work was performed in air, resulting in low quality factors of the cantilever, i.e., 120 and 500 on the first and second mode, respectively. Another interesting experimental approach suited for low quality factor cantilevers is based on narrowband intermodulation atomic force microscopy. ${ }^{6}$

In our work, we developed a non-contact bimodal magnetic force microscopy technique which can take advantage of the higher sensitivities provided by high quality factor cantilevers. It is optimally suited for operation in vacuum or UHV for highest sensitivity and thus best spatial resolution.

Each spatial Fourier component of the magnetic stray fields decays exponentially with increasing distance $z$ from the surface of a magnetic sample, $H_{z}(\mathbf{k}, z)=H_{z}(\mathbf{k}, 0) \cdot \exp (-k z)$, where $H_{z}(\mathbf{k}, 0)$ is the stray field at the surface of the sample,

\footnotetext{
${ }^{\text {a) }}$ Author to whom correspondence should be addressed. Electronic mail: johannes.schwenk@empa.ch.
}

and $\mathbf{k}=\left(k_{x}, k_{y}\right)$ with $k=2 \pi / \lambda$, where $\lambda$ is the spatial wavelength. ${ }^{7}$ Given a sufficiently sharp tip with an appropriately thin magnetic coating, high resolution imaging thus requires small tip-sample distances, correspondingly small oscillation amplitudes, and a high sensitivity for small forces or force derivatives.

We used a cantilever from Team Nanotec GmbH with an ISC (Improved Super Cone) high aspect ratio tip coated with about $8 \mathrm{~nm}$ Co. Such a thin magnetic coating leads to a correspondingly small magnetic moment. This is advantageous if soft magnetic samples are measured, but requires an excellent measurement sensitivity to detect the small magnetic signals arising from smaller spatial wavelengths of the stray field. The thermodynamic limit for the minimally measurable force derivative on the nth cantilever oscillation mode is given by ${ }^{8}$

$$
\left.\frac{\partial}{\partial z} F_{z}\right|_{\min }[\mathrm{N} / \sqrt{\mathrm{Hz}}]=\frac{1}{A_{n}} \sqrt{\frac{4 k_{B} T k_{n}}{Q_{n} \omega_{n}}} .
$$

The force constant $k_{n}$ and the resonance frequency $\omega_{n}$ of the nth mode relate to those of the first mode as

$$
k_{n}=k_{1} \cdot\left[\frac{\alpha_{n}}{\alpha_{1}}\right]^{4} \quad \text { and } \quad \omega_{n}=\omega_{1} \cdot\left[\frac{\alpha_{n}}{\alpha_{1}}\right]^{2},
$$

with $\alpha_{i}=\{1.8750,4.6941,7.8548,10.9955, \ldots\}$. $^{8}$

According to Eqs. (1) and (2) the sensitivity of a higher mode $n$ is lower than for the first mode by $\alpha_{n} / \alpha_{1} \cdot \sqrt{Q_{1} / Q_{n}}$. The first mode is hence better suited for the measurement of the small magnetic forces. In order to obtain higher quality factors, our MFM (a modified Nanoscan hrMFM) with two phase-locked loops (PLLs) (Fig. 1) is operated under vacuum conditions. The first mode force constant of the cantilever, $k_{1}=0.996 \mathrm{~N} / \mathrm{m}$ was calculated from the measured first mode resonance frequency, $f_{1}=53^{\prime} 059.9 \mathrm{~Hz}$, the length $l=225 \mu \mathrm{m}$ and the width $w=35 \mu \mathrm{m}$. The second mode force constant $k_{2}=39.1 \mathrm{~N} / \mathrm{m}$ was obtained from Eq. (2), and the second mode resonance frequency was measured, $f_{2}=327^{\prime} 850 \mathrm{~Hz}$. In vacuum, the first and second mode quality factors were $Q_{1}=40^{\prime} 268$ and $Q_{2}=17^{\prime} 205$, respectively. Such high quality factors do not permit the excitation of the cantilever at a user-selected fixed frequency, but require PLLs to drive the first and second mode on their respective resonance frequencies (Fig. 1). The amplitude response of 


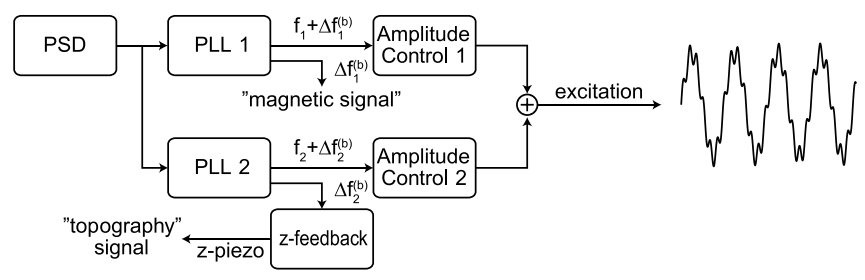

FIG. 1. Schematic of the dual-PLL system required for bimodal oscillation of high quality factor cantilevers. PLL 1 tracks the first flexural oscillation mode resonance frequency $f_{1}$ of the cantilever. The output amplitude is adjusted by a PI feedback (Amplitude Control 1) to keep the (first mode) oscillation amplitude constant, e.g., at $12 \mathrm{~nm}$. The shift of the first mode resonance frequency, $\Delta f_{1}^{(b)}$ predominantly reflects the longer-ranged magnetic part of the tip-sample interaction. PLL 2 tracks the second flexural oscillation mode resonance frequency $f_{2}$ of the cantilever. The PI feedback Amplitude Control 2 keeps the second mode amplitude constant, e.g., at $0.27 \mathrm{~nm}$. At such a small amplitude, the second mode resonance frequency shift $\Delta f_{2}^{(b)}$ predominantly arises from the van der Waals part of the tip-sample interaction, which depends on the tip-sample distance but not on the micromagnetic details of the sample. $\Delta f_{2}^{(b)}$ and thus the local tip-sample distance is kept constant by the z-feedback. Its output then reflects the topography of the sample.

the deflection detector of our MFM was calibrated by a thermal noise analysis. ${ }^{9}$ The z-feedback adjusts the tip-sample distance to keep the second mode frequency shift constant.

As outlined by Giessibl et al., ${ }^{10}$ the frequency shift of a cantilever of stiffness $k$ oscillating in a single mode with an amplitude $A$ in a force field derivative $\frac{\partial}{\partial z} F_{z}(z)=k_{t s}(z)$ becomes

$$
\Delta f\left(z_{l t p}\right)=\frac{f_{0}}{2 k} \frac{2}{\pi A^{2}} \int_{-A}^{A} k_{t s}\left(z_{l t p}+A-q\right) \cdot \sqrt{A^{2}-q^{2}} d q
$$

where $z_{l t p}$ is the tip-sample distance at the lower turning point, $f_{0}$ is the resonance frequency of the cantilever far away from the surface, and $k_{t s}$ is the local stiffness of the tip-sample interaction. In the limit of small amplitudes, the frequency shift becomes proportional to the stiffness of the tip-sample interaction force

$$
\Delta f(z)=-\frac{f_{0}}{2 k} \cdot k_{t s}(z)
$$

This approximation is valid as long as $k_{t s}$ is approximately constant over the z-range spanned by the oscillating tip. At small tip-sample distances the z-derivative of the van der Waals force is usually much stronger than that of the magnetic force. In that case, a frequency shift measured with a cantilever oscillating with a small amplitude at a small tipsample distance is dominated by the non-magnetic van der Waals force. At considerably larger amplitudes, the small amplitude approximation no longer holds and Eq. (3) must be used to calculate the frequency shift. In essence, Eq. (3) averages $k_{t s}(z)$ over time. The weaker but longer-ranged magnetic stiffness curve dominates that of the stronger but shorter-ranged van der Waals stiffness curve. The long range magnetic force then becomes the dominant contribution to the measured frequency shift. Note that these observations hold independently of the cantilever oscillation mode. So, for the same oscillation amplitude, the frequency shifts of the n-th mode simply scale to those of the first mode as $\Delta f_{n}=\Delta f_{1} \cdot \alpha_{1}^{2} / \alpha_{n}^{2}$.
In bimodal operation conditions with large $A_{1} / A_{2}$-ratios, the frequency shift versus distance curve $\Delta f_{2}^{(b)}\left(z_{l t p}\right)$ has a distance dependence closely resembling what would be measured without the first mode oscillation amplitude $\left(\Delta f_{2}\left(z_{l t p}\right)\right)$. However, the frequency shift values are smaller at all distances. ${ }^{11}$ The second mode bimodal frequency shift $\Delta f_{2}^{(b)}\left(z_{l t p}\right)$ is thus also dominated by the short-range van der Waals force. Consequently, if $\Delta f_{2}^{(b)}$ is kept constant by the (fast) z-feedback, the output of the latter corresponds to the topography of the sample. The bimodal frequency shift of the first mode, $\Delta f_{1}^{(b)}$, is not affected by the presence of the small-amplitude second mode oscillation because the cantilever oscillation modes are not harmonic. ${ }^{411}$ Hence, $\Delta f_{1}^{(b)}$ reflects the long range magnetic force.

Fig. 2 summarizes MFM results obtained with different ratios of the first to second mode amplitudes in bimodal operation on a $\mathrm{Pt}(5 \mathrm{~nm}) /[\mathrm{Co}(0.4 \mathrm{~nm}) / \mathrm{Pt}(0.7 \mathrm{~nm})]_{15} \mathrm{Pt}(1 \mathrm{~nm}) / \mathrm{SiO}_{2}^{\alpha x} / \mathrm{Si}$ sample with perpendicular magnetic anisotropy. The second mode frequency shift $\Delta f_{2}^{(b)}$ was kept constant at $-6.8 \mathrm{~Hz}$ for all data shown here. An excellent separation of the magnetic and topographic signal is obtained for an $A_{1} / A_{2}$-ratio of 44 (Figs. 2(a)-2(c)). The first bimodal frequency shift $\Delta f_{1}^{(b)}$ data (Fig. 2(a) shows the up/down domain structure typical for such a sample, while the output of the z-feedback (Fig. 2(b) operating on the second bimodal frequency shift $\Delta f_{2}^{(b)}$ reflects the topography of the sample. The instrumental drift of the sample's z-position becomes apparent as the image scanned from the

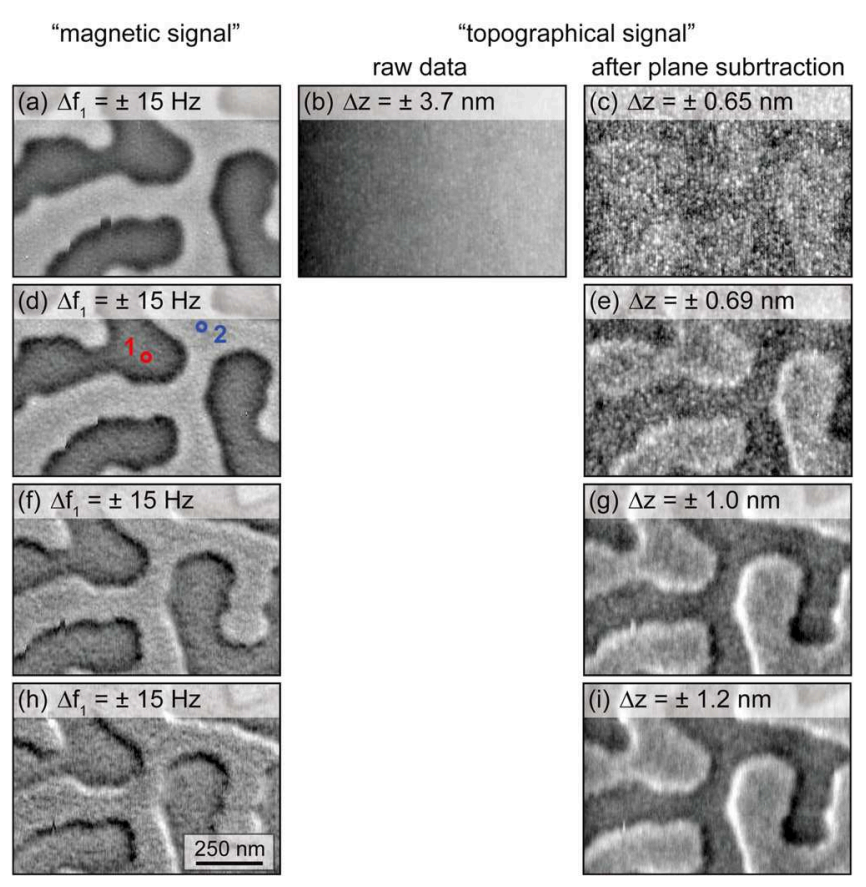

FIG. 2. Images (a) to (c) were measured with an $A_{1}=12 \mathrm{~nm}$. (a) magnetic image (first mode frequency shift). (b) topography image revealing the drift of the tip-sample distance (the image was scanned from bottom to top and left to right) and the grain structure. The latter becomes better visible in image (c) where the drift plane has been subtracted. Magnetic contrast and topography images for $A_{1}$ equal to $6 \mathrm{~nm}, 3 \mathrm{~nm}$, and $1.5 \mathrm{~nm}$ are shown in panels (d) and (e), (f) and (g), and (h) and (i), respectively. The amplitude of the second mode was kept $A_{2}=0.27 \mathrm{~nm}$ for all measurements. Note that the $\Delta f$ or $\Delta z$ values given in the images represent the displayed grey scales, and not the actual extrema of the image contrast. Thus, corresponding images can be directly compared. The red and blue circles in panel (d) mark the acquisition locations of the frequency-distance curves shown in Fig. 3. 
(a)

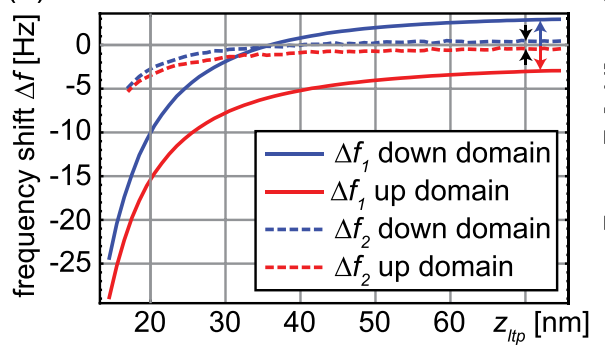

(b)

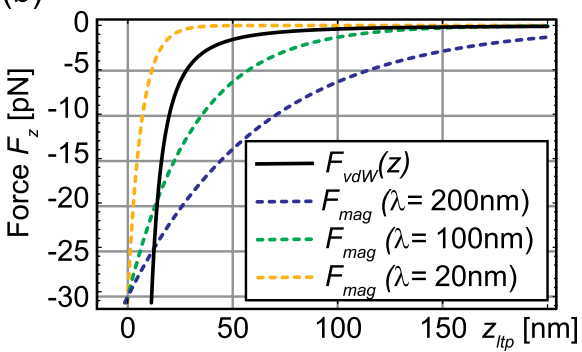

FIG. 3. (a) First and second mode frequency distance curves measured with $A_{1}=6 \mathrm{~nm}$ and $A_{2}=0.27 \mathrm{~nm}$ at the locations marked with a red and blue circle in Fig. 2. (b) van der Waals force as a function of distance extracted from the first mode frequency shift curves shown in (a). Magnetic force as a function of distance for different spatial wavelengths. Note that at much larger tip-sample distance (not shown here) all $\Delta f$-curves will approach zero and all magnetic contrast vanishes.

bottom to the top and from the left to the right appears darker at the left and brighter at the right. After subtracting the "drift-plane," the topography image, Fig. 2(c), reveals the granular structure of the polycrystalline sample with grain sizes around $20 \mathrm{~nm}$ and height variations of $\pm 0.65 \mathrm{~nm}$. The magnetic domains are faintly visible, however, (a contrast of about $0.1 \mathrm{~nm}$ ) indicating that the separation of magnetic and van der Waals forces is not perfect.

Reducing the $A_{1} / A_{2}$-ratio from the initial 44 down to 22 (Figs. 2(d) and 2(e)), 11 (Figs. 2(f) and 2(g)), and 5.6 (Figs. 2(h) and 2(i)), the magnetic contrast in the z-feedback output images increases. To understand this, note that the bimodal frequency shift $\Delta f_{2}^{(b)}$ is smaller than the unimodal frequency shift $\Delta f_{2}$ because in bimodal operation the $\Delta f_{2}^{(b)}$ is averaged over the tip-sample distance range spanned by the first mode oscillation amplitude $A_{1}$. With smaller amplitude $A_{1}$ this averaging of the second mode frequency shift occurs over a smaller z-range. In that case $\Delta f_{2}^{(b)}$ is larger, closer to the $\Delta f_{2}$ that would be obtained in unimodal operation. For the data shown in Fig. 2, the z-feedback setpoint was kept constant at $-6.8 \mathrm{~Hz}$ for all selected amplitudes $A_{1}$. Consequently, the $\mathrm{z}$-feedback will increase the tip-sample distance if the first mode amplitude $A_{1}$ is reduced. At larger tip-sample distances the contribution of the longer-ranged magnetic forces to the frequency shift is more prominent, and the magnetic domain contrast becomes more apparent (Figs. 2(e)-2(i)) in the $\mathrm{Z}$-feedback output data. But since the $\mathrm{z}$-feedback will partially have corrected for the spatially dependent magnetic forces, the magnetic contrast visible in the $\Delta f_{1}^{(b)}$-images (Figs. 2(d)-2(h)) is reduced.

We also acquired frequency shift versus distance curves simultaneously for the first and second mode, in the middle of a black and of a white domain (Fig. 2(d), points 1 and 2). We used oscillation amplitudes $A_{1}=6 \mathrm{~nm}$ and $A_{2}=0.27 \mathrm{~nm}$. At $z_{l t p}=70 \mathrm{~nm}$, the contrast between the $\Delta f_{1}^{(b)}$ values obtained on the white (wd) and black domain (bd) corresponds to $5.9 \mathrm{~Hz}$ (blue-red arrow in Fig. 3(a). $70 \mathrm{~nm}$ away from the sample, the change of the force $\mathrm{z}$-derivative over the tip's oscillation trajectory with the chosen oscillation amplitudes can then be neglected. Therefore, the small amplitude approximation, i.e., Eq. (4) holds and $\Delta f_{2}^{(b)}(\mathrm{wd})$ $-\Delta f_{2}^{(b)}(\mathrm{bd}) \approx\left[\Delta f_{1}^{(b)}(\mathrm{wd})-\Delta f_{1}^{(b)}(\mathrm{bd})\right] \cdot\left(\alpha_{1} / \alpha_{2}\right)^{2}=0.94 \mathrm{~Hz}$ agreeing well with the experimental value of $0.89 \mathrm{~Hz}$ (black arrows in Fig. 3(a). Because the second mode amplitude is small $\left(A_{2}=0.27 \mathrm{~nm}\right)$, the second mode frequency shift, $\Delta f_{2}^{(b)}$ is dominated by the van der Waals force at small tip-sample distances. Hence, the tip-sample distance required to correct between a magnetically attractive and -repulsive signal is extremely small. Assuming that the magnetic forces at these two points have equal magnitudes but opposite signs, the average of the two $\Delta f_{1}^{(b)}$-curves is solely caused by the van der Waals interaction. Using the matrix inversion method described by Welker et al., ${ }^{12}$ the distance dependence of the van der Waals force $F_{v d W}\left(z_{l t p}\right)$ (depicted in Fig. 3(b)) can be extracted from the average frequency shift $\Delta f_{1, v d W}^{(b)}\left(z_{l t p}\right)$. A model counterpart for this experimental van der Waals force is $F_{v d w}(z)=-c /\left(z-z_{\text {offs. }}\right)^{2}$ with $c=3.87 \cdot 10^{-27} \mathrm{Nm}^{2}$ (Fig. 3(b), solid black line). The constant $z_{\text {offs. }}$ is required because the zero-point of the z-scale in Fig. 3(a) is a priori unknown and an absolute zero-point can be defined to obtain $z_{\text {offs. }}=0$. For comparison, we also plot exponentially decaying magnetic forces $F_{\text {mag }}(z)=F_{0} \cdot \exp (-k z)$ for spatial wavelengths $\lambda=2 \pi / k=200,100$, and $20 \mathrm{~nm}$. The constant $F_{0}$ has been adjusted so that the $200 \mathrm{~nm}$ plot reproduces the experimentally determined contrast $\Delta f_{1}^{(b)}(w d)-\Delta f_{1}^{(b)}(b d)=5.9 \mathrm{~Hz}$ at a tip-sample distance of $70 \mathrm{~nm}$. Notice that the magnetic force arising from magnetization patterns with $20 \mathrm{~nm}$ spatial wavelength has a decay length similar to that of the van der Waals force. High-resolution MFM based on bimodal techniques is still possible, however, if the van der Waals force is much larger than the magnetic force arising from magnetic field features of small spatial wavelengths, i.e., if $\Delta f_{2, \text { mag }}^{(b)}(\Delta z) \ll \Delta f_{2, v d W}^{(b)}(\Delta z)$. Under this condition, a change of the magnetic force would be compensated by a negligible change of the tip-sample distance. Therefore, the $\mathrm{z}$-feedback image still reflects the topography and the $\Delta f_{1}^{(b)}$ image shows the magnetic image.

For high-resolution imaging (Fig. 4), the tip-sample distance must be kept small. The latter can be estimated from the frequency distance curve (Fig. 3(a) and the z-feedback
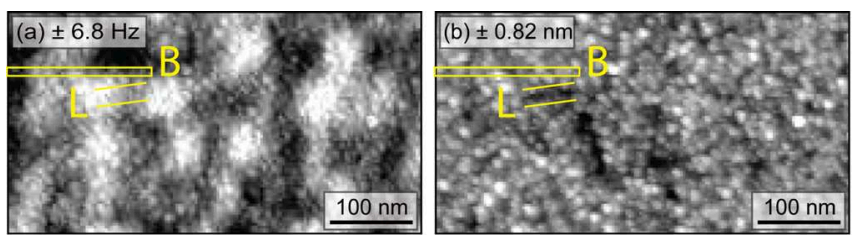

FIG. 4. (a) High resolution magnetic image of a modern hard-disc recorded with $A_{1}=12 \mathrm{~nm}$. (b) Topography measured simultaneously with the magnetic signal with $A_{2}=0.27 \mathrm{~nm}$. The grain structure is nicely visible in both images. The $10 \mathrm{~nm}$ high yellow box serves as a guide for the eye to judge the lateral resolution of the MFM image. 
set-point $\Delta f_{2}^{(b)}=-6.8 \mathrm{~Hz}$ that was used for the data shown in Fig. 4. A lower turning point tip-sample distance of $7.6 \mathrm{~nm}$ is found. Note, however, that the proper function of the z-feedback requires a low noise $\Delta f_{2}^{(b)}$-signal at a reasonable measurement bandwidth. Using Eqs. (1) and (4) we find a $\Delta f_{2}$-noise of $0.66 \mathrm{~Hz}$ in a $100 \mathrm{~Hz}$ bandwidth for the high quality factor $Q_{2}=17^{\prime} 205$ obtained in vacuum for $A_{2}=0.27 \mathrm{~nm}$. This noise must be compared with the second mode frequency shift obtained under bimodal operation. Hence, for typical quality factors obtained in air, a stable operation of the z-feedback on $\Delta f_{2}^{(b)}$, at a reasonable measurement bandwidth becomes challenging. This limits the application of bimodal MFM as proposed here to vacuum operation and reasonably small scan-ranges. To summarize, we have presented a non-contact bimodal magnetic force microscopy technique capable of obtaining highest resolution and sensitivity for magnetic stray fields under vacuum conditions. However (as is also the case for conventional, lift-mode operation), the topography will be partly visible in the first mode frequency shift and may easily be mistaken for small magnetic features. This is because magnetic forces will vary as the tip follows local topographical features. For example, the grain structure visible inside the white bits in the magnetic image Fig. 4(a) is not caused by variations of the local magnetic structure, but arises from local changes of the average tip-sample distance. However, in Fig. 4 in some locations (see for example the yellow box B with a height of $10 \mathrm{~nm})$ the magnetic contrast changes from bright to dark from one grain to the next, although adjacent grains have the same topographical height. Such a contrast change must be of magnetic origin. Further, in some locations (see the parallel lines L) topographical features are visible that do not appear in the magnetic image. This indicates that a lateral resolution on the length scale of the grains, i.e., smaller than $10 \mathrm{~nm}$ could be obtained.

Support from Nanoscan and the financial support of CCMX, the Swiss National Science Foundation, and Empa is hereby gratefully acknowledged. We thank Eric Hudson for his valuable suggestions concerning this manuscript.

${ }^{1}$ I. Schmid, M. A. Marioni, P. Kappenberger, S. Romer, M. ParlinskaWojtan, H. J. Hug, O. Hellwig, M. J. Carey, and E. E. Fullerton, Phys. Rev. Lett. 105, 197201 (2010).

${ }^{2}$ S. Hosaka, A. Kikukawa, Y. Honda, H. Koyanagi, and S. Tanaka, Jpn. J. Appl. Phys., Part 2 31, L904 (1992).

${ }^{3}$ R. Giles, J. P. Cleveland, S. Manne, P. K. Hansma, B. Drake, P. Maivald, C. Boles, J. Gurley, and V. Elings, Appl. Phys. Lett. 63, 617 (1993).

${ }^{4}$ J. W. Li, J. P. Cleveland, and R. Proksch, Appl. Phys. Lett. 94, 163118 (2009).

${ }^{5}$ T. R. Rodriguez and R. Garcia, Appl. Phys. Lett. 84, 449 (2004).

${ }^{6}$ D. Forchheimer, D. Platz, E. A. Tholén, and D. B. Haviland, Appl. Phys. Lett. 103, 013114 (2013).

${ }^{7}$ E. Meyer, H. J. Hug, and R. Bennewitz, Scanning Probe Microscopy 4.2.2 (Springer, 2003).

${ }^{8}$ H.-J. Butt and M. Jaschke, Nanotechnology 6, 1 (1995).

${ }^{9}$ M. J. Higgins, R. Proksch, J. E. Sader, M. Polcik, S. Mc Endoo, J. P. Cleveland, and S. P. Jarvis, Rev. Sci. Instrum. 77, 013701 (2006).

${ }^{10}$ F. J. Giessibl, H. Bielefeldt, S. Hembacher, and J. Mannhart, Ann. Phys. Leipzig 10, 887 (2001).

${ }^{11}$ S. Kawai, T. Glatzel, S. Koch, B. Such, A. Baratoff, and E. Meyer, Phys. Rev. Lett. 103, 220801 (2009).

${ }^{12}$ J. Welker, E. Illek, and F. J. Giessibl, Beilstein J. Nanotechnol. 3, 238 (2012). 